\title{
Comparing the performance of hyperbolic and circular rod quadrupole mass spectrometers with applied higher order auxiliary excitation
}

\author{
D.J. Gershman a,*, B.P. Block ${ }^{\mathrm{a}}$, M. Rubin ${ }^{\mathrm{a}}$, M. Benna ${ }^{\mathrm{b}}$, P.R. Mahaffy ${ }^{\mathrm{b}}$, T.H. Zurbuchen ${ }^{\mathrm{a}}$ \\ a Department of Atmospheric, Oceanic and Space Sciences, University of Michigan, Ann Arbor, MI, USA \\ ${ }^{\mathrm{b}}$ Solar Systems Exploration Division, NASA Goddard Space Flight Center, Greenbelt, MD, USA
}

\section{A R T I C L E I N F O}

\section{Article history:}

Received 21 February 2012

Received in revised form 24 March 2012

Accepted 24 March 2012

Available online 2 April 2012

\section{Keywords:}

Quadrupole mass spectrometer

Auxiliary excitation

Spaceborne mass spectrometry

\begin{abstract}
A B S T R A C T
This work applies higher order auxiliary excitation techniques to two types of quadrupole mass spectrometers (QMSs): commercial systems and spaceborne instruments. The operational settings of a circular rod geometry commercial system and an engineering test-bed for a hyperbolic rod geometry spaceborne instrument were matched, with the relative performance of each sensor characterized with and without applied excitation using isotopic measurements of $\mathrm{Kr}^{+}$. Each instrument was operated at the limit of the test electronics to determine the effect of auxiliary excitation on extending instrument capabilities. For the circular rod sensor, with applied excitation, a doubling of the mass resolution at $1 \%$ of peak transmission resulted from the elimination of the low-mass side peak tail typical of such rod geometries. The mass peak stability and ion rejection efficiency were also increased by factors of 2 and 10 , respectively, with voltage scan lines passing through the center of stability islands formed from auxiliary excitation. Auxiliary excitation also resulted in factors of 6 and 2 in peak stability and ion rejection efficiency, respectively, for the hyperbolic rod sensor. These results not only have significant implications for the use of circular rod quadrupoles with applied excitation as a suitable replacement for traditional hyperbolic rod sensors, but also for extending the capabilities of existing hyperbolic rod QMSs for the next generation of spaceborne instruments and low-mass commercial systems.
\end{abstract}

(c) 2012 Elsevier B.V. All rights reserved.

\section{Introduction}

For the past 50 years, the quadrupole mass spectrometer (QMS) has been the instrument of choice for high quality neutral gas composition measurements in both the commercial realm [1] and for spaceborne applications [2-5]. Commercial sensors typically employ more easily manufactured circular rods, but can suffer from degraded peak shapes and difficulties in attaining high resolution measurements [6], though proper sizing of circular rods can help to minimize these effects [7].

Spaceborne instruments, on the other hand, have relied on hyperbolic geometry, as these rods provide a near-ideal quadrupole electric field. However, it can be extremely challenging and expensive to manufacture and align such rod sets. In their traditional mode of operation, these sensors are perhaps at the limits of their capabilities, as newly developed ion sources with optimal beam design virtually eliminate affects of fringing fields, yet the highest attained mass range for modern space instruments extends only to $500 \mathrm{Da}$ [8]. However, for mass spectrometers that are part of future planetary and cometary missions, mass ranges beyond 1000 Da will

\footnotetext{
* Corresponding author.

E-mail address: djgersh@umich.edu (D.J. Gershman).
}

be required in order to measure complex organics and volatiles that are currently at the center of scientific interest, as they relate to the investigation of sources and processes of life in space [9]. In order to use well-proven QMS technology for the next generation of spaceborne mass spectrometers, new techniques that improve overall instrument performance must be developed and tested. Auxiliary excitation techniques will be shown here to accomplish such an improvement.

Stable ion trajectories in a QMS are created by applying two voltages to the quadrupole rods: an RF waveform at a frequency typically in the $\mathrm{MHz}$ range, and a DC potential. A subset of the possible RF and DC amplitudes results in stable trajectories for a given species of ion. Mass peaks corresponding to increasing ion mass-tocharge $(\mathrm{m} / \mathrm{z})$ ratios can be produced by increasing the $\mathrm{RF} / \mathrm{DC}$ voltage amplitudes while keeping their ratio constant, forming a voltage scan line. The slope of this scan line will determine the width, i.e., the resolution, of the mass peak [6]. In order to maximize the sensitivity of the instrument, quadrupoles are often tuned to unity mass resolution such that the scan line slope is set to increase with increasing $\mathrm{m} / \mathrm{z}$ ratios [10]. However, for a measurement confined to a small mass range, a constant scan line slope is appropriate.

Auxiliary excitation techniques apply an additional RF component Cting resonances in the system that split the original solid RF/DC stability region into small islands of stability. Operation 
with voltage scan lines through the tips of these islands has been shown through experimental work [11-15] to significantly improve the performance of circular rod quadrupole mass spectrometers by improving peak shapes, abundance sensitivity, and mass resolution. Auxiliary frequencies are typically set to be very low $[14,15]$, or near to, but not identical to that of the fundamental RF signal [13]. These techniques cannot easily be applied to space instrumentation due to the high degree of precision required to drive and control such a small auxiliary signal amplitude. Furthermore, since the quadrupole rods typically act as part of a resonant tank circuit that enables more power efficient operation, inserting a signal off-resonance, even at small amplitudes, requires large amounts of driving current, a key resource on spacecraft [16]. Even for terrestrial applications, large required driving currents can prohibit the creation of very small islands of stability such that it has been easier to create larger islands of stability and operate scan lines through their tips.

Higher order auxiliary excitation techniques [16,17] drive the quadrupole rods at a frequency nearly twice that of the fundamental frequency. These higher order techniques provide the same advantages of previously demonstrated auxiliary excitation driven at lower frequencies, but allow improved dynamic range of the sensed auxiliary signal amplitude. When used in conjunction with a multi-resonant tank circuit [16], the quadrupole rods can be driven efficiently at both the fundamental RF and auxiliary frequencies, enabling auxiliary excitation for power-limited applications such as space-flight. Furthermore, larger excitation amplitudes permitted by this circuit enables operation of voltage scan lines through the center of small upper stability islands, providing more stable peak shapes than voltage scan lines passing through the island tips [16], an improvement important for space and commercial applications alike.

The advantages of higher order auxiliary excitation techniques when applied to circular rod QMSs at lower resolution $(\sim 100)$ have recently been experimentally verified using measurements of $\mathrm{Ar}^{+}$ [16]. Numerical simulations have mapped out the set of practical instrument operating points [17] as well as predicted that these techniques should improve the performance of sensors with ideal hyperbolic rod geometry $[17,18]$, but these advantages have yet to be experimentally verified. This work will serve as the first experimental demonstration of higher order auxiliary excitation techniques in a hyperbolic geometry QMS. Here, higher order auxiliary excitation is applied to two sets of rod sets (Section 2) with circular and hyperbolic rod geometry respectively, whose operating characteristics have been well matched. Isotopic measurements of $\mathrm{Kr}^{+}$(Section 3) are used as a baseline for gauging sensor performance.

\section{Experimental setup}

A Field Programmable Gate Array (FPGA)-based QMS voltage control system and multiresonant tank circuit described in detail by Gershman et al. [16] were modularized and interfaced with two existing quadrupoles as drop-in replacements for their respective drive electronics. The first, a commercial Pfeiffer QMG422 circular rod system, was previously used to demonstrate the improved performance using high order auxiliary excitation with measurements of $\mathrm{Ar}^{+}$[16]. For this sensor, with a rod radius to spacing ratio of 1.20, a significant low-mass peak tail is expected [8] and in fact observed in nominal sensor operation [16]. The second quadrupole was a space-flight quality hyperbolic rod system, the engineering test bed for the Sample Analysis at Mars (SAM) instrument [9] on the Mars Science Laboratory (MSL) mission. The physical characteristics as well as timing and operating parameters for each sensor are summarized in Table 1 with details on their selection described throughout this section.
Table 1

Characteristics of QMS sensors used in this study.

\begin{tabular}{lll}
\hline & Hyperbolic & Circular \\
\hline Physical characteristics & & \\
Rod length $(l)(\mathrm{mm})$ & 150 & 300 \\
Rod spacing $\left(r_{o}\right)(\mathrm{mm})$ & 5.08 & 6.7 \\
$\quad$ Rod radius $(\mathrm{mm})$ & $\mathrm{N} / \mathrm{A}$ & 8 \\
Operating point & & \\
Fundamental frequency $(\Omega / 2 \pi)(\mathrm{MHz})$ & 1.40 & 1.06 \\
Excitation frequency $(\omega / 2 \pi)(\mathrm{MHz})$ & 2.6834 & 2.0317 \\
RF amplitude per amu/e $(\mathrm{V} /(\mathrm{m} / \mathrm{z}))(\mathrm{V} /(\mathrm{amu} / \mathrm{e}))$ & 3.68 & 3.67 \\
Nominal rod bias potential $\left(\varphi_{b}\right)(\mathrm{V})$ & -2 & +70 \\
Nominal source exit potential $\left(\varphi_{s}\right)(\mathrm{V})$ & 0 & 0 \\
$\quad$ Nominal ion energy from source $E_{i, s}(\mathrm{~V})$ & $1 \pm 0.5$ & $+80 \pm 5$ \\
Accumulations & & \\
Steps per amu/e & 20 & 64 \\
Step dwell time $(\mathrm{ms})$ & 100 & 0 \\
Step accumulation time $(\mathrm{ms})$ & 50 & 16 \\
\hline
\end{tabular}

In order to obtain a meaningful comparison between different QMS sensors, the operating characteristics of each must be matched as well as possible, though there will inevitably be some discrepancy between their observed performances due to differences in test setups. Nevertheless, matching the important non-dimensional numbers across systems ensures maximum consistency. The dimensionless numbers of a quadrupole sensor, $a$, $q$, and $\xi$, arise from the equations of motion of an ion in an ideal quadrupole field with added auxiliary excitation [18],

$$
\begin{gathered}
\frac{d^{2} x}{d \xi^{2}}+(a-2 q \cos (2 \xi)) x-2 q^{\prime} \cos \left(2 \xi \frac{\omega}{\Omega}\right) x=0 \text { and } \\
\frac{d^{2} y}{d \xi^{2}}-(a-2 q \cos (2 \xi)) y+2 q^{\prime} \cos \left(2 \xi \frac{\omega}{\Omega}\right) y=0
\end{gathered}
$$

with

$a \equiv \frac{8 e U}{(m / z) r_{o}^{2} \Omega^{2}}, \quad q \equiv \frac{4 e V}{(m / z) r_{o}^{2} \Omega^{2}}, \quad$ and $\quad \xi \equiv \frac{\Omega t}{2}$.

Here, $x$ and $y$ are the transverse position coordinates of an ion in a 2D quadrupole field, $U$ and $V$ are the DC and RF voltage amplitudes pole to ground, respectively, $r_{o}$ is the rod spacing, $\Omega$ is the fundamental operating frequency, $\omega$ is the auxiliary frequency, $e$ is the unit of an electric charge, $m / z$ is the mass-to-charge ratio, and $t$ is the time. $q^{\prime}$ is the relative strength of the excitation defined by $q^{\prime} \equiv q V_{e x} / V$ where $V_{e x}$ is the excitation voltage amplitude.

The matching of $a$ and $q$ will be accomplished through appropriate frequency selection and the use of identical drive electronics for each sensor. $\xi$ will be matched across sensors through proper selection of ion axial energies. Finally, the dynamic range of the detected ion signal will be adjusted to ensure similar counting statistics for each QMS.

The experimental setup here has the same characteristics and limitations as those described in Gershman et al. [16]. Consequently, we will focus on low-resolution $((m / z) / \Delta(m / q) \sim 100)$ measurements. However, these measurements will be sufficient to show good agreement with previous numerical simulations and illustrate the advantages of auxiliary excitation for both rod geometries. For each sensor, the multiresonant tank circuit was tuned to obtain a ratio of auxiliary to fundamental frequencies $(\omega / \Omega)$ of 1.9167. Such precise frequency selection is enabled through use of digitally synthesized signals by an FPGA. This excitation frequency will create an upper stability island that will produce a mass peak with resolution on the order of 100 when a voltage scan line, whose required precision is at the limit of the developed electronics, is swept through the island center. Only by examining the operation at this limit can the effectiveness of auxiliary excitation techniques on extending the capabilities of QMS sensors be determined. 


\subsubsection{Matching of $a$ and $q$}

For a typical high-resolution measurement, operation of scan lines through the tip of the stability region requires voltages such that $a \approx 0.237$ and $q \approx 0.706$. While it might make intuitive sense to operate each quadrupole at identical frequencies, the differing physical dimensions of each sensor will result in different required voltages to achieve stable trajectories for a particular ion. The driving electronics will control the accuracy and precision of the instrument operating point, and consequently, the trajectories of analyzed ions. Following Eq. (2), if the $U /(\mathrm{m} / \mathrm{z})$ and $V /(\mathrm{m} / \mathrm{z})$ ratios are matched across rod sets, noise in the voltage-control system will have similar effects on ion trajectories for each sensor, and differences in performance should be dominated by differences in the physical properties of the rods, rather than electronic effects since these voltages scale as $r_{o}^{2} \Omega^{2}$.

Since all frequency components are digitally generated using an FPGA, a change in operating frequency is readily accomplished. Therefore, given the field radius, $r_{o}$, of a rod set, the operating frequencies can be adjusted to obtain the same $V /(m / z)$ ratios. For $\Omega / 2 \pi=1.06 \mathrm{MHz}$ and $r_{0}=6.7 \mathrm{~mm}$ for the circular rod set, and $\Omega / 2 \pi=1.4 \mathrm{MHz}$ and $r_{0}=5.08 \mathrm{~mm}$ for the hyperbolic rod set, the $V /(\mathrm{m} / \mathrm{z})$ values are well matched at $3.67 \mathrm{~V} /(\mathrm{amu} / \mathrm{e})$ and $3.68 \mathrm{~V} /(\mathrm{amu} / \mathrm{e})$ respectively, as shown in Table 1 . Although tuning adjustments were made to the multiresonant tank circuit to accommodate each sensor's rod capacitance, the overall drive electronics were identical for each quadrupole.

\subsubsection{Matching of the number of RF cycles in the field}

Assuming constant ion axial energy $E$, $\xi$ from Section 2 can be written as a function of the rod length, ion energy, and ion $m / z$ ratio, as,

$\xi=\frac{\Omega l}{2 \sqrt{2 e E /(m / z)}}$.

The number of RF cycles ( $n$ ) experienced by an ion traversing the length of the quadrupole rods is commonly used instead of $\xi$ to describe instrument behavior [6]. The two quantities are related to one another by $\xi=\pi n$. The ideal quadrupole, which is infinitely long, has an infinite number of RF cycles to filter out unwanted ions, creating perfectly sharp stability boundaries. This filtering process results in high-resolution mass peaks obtained with scan lines passing near the tip of the stability region. However, all practical instruments have finite length rods, and consequently, only a finite number of RF cycles to reject unwanted ions. If $n$ is too low, a significant number of unwanted ions remain unfiltered, contaminating adjacent mass peaks. When the resolution is limited by the number of RF cycles, rather than rod quality or other factors, the maximum attainable mass resolution of a QMS scales as $n^{2}$ [6].

In order to compare the performance of different rod sets, the $n$-values of ions should be well matched. For a given quadrupole, $l$ is fixed, and the operating frequency $\Omega$ is set as described in Section 2.1.1. Therefore, from Eq. (3) the ion axial energy $(E)$ must be adjusted in order to obtain a given $n$-value. The available range of ion axial energies is typically set by the design of the ion source. However, the quadrupole rods themselves can be biased with a potential with respect to the ion source exit aperture. The final ion axial energy $(E)$ is approximated by the potential difference between the rods $\left(\varphi_{b}\right)$ and the exit aperture of the source $\left(\varphi_{s}\right)$ subtracted from the ion energy produced by the source $\left(E_{i, s}\right)$, such that,

$E \approx E_{i, s}-\left(\phi_{b}-\phi_{s}\right)$
The approximation of Eq. (4) neglects fringing field and field penetration effects between the rods and the exit aperture of the source, as well as the spread of produced ion energies.

For the circular rod set, ions were created with energies approximately $80 \pm 5 \mathrm{eV}$. A $+70 \mathrm{~V}$ to $+40 \mathrm{~V}$ rod bias range is used to obtain ions between $n \approx 35$ and $n \approx 70 \mathrm{RF}$ cycles. For the hyperbolic rod set, ions were created at approximately $1 \pm 0.5 \mathrm{eV}$. Therefore, a rod bias between $-2 \mathrm{~V}$ and $-16 \mathrm{~V}$ is used to obtain ions between $n \approx 35$ and $n \approx 80 \mathrm{RF}$ cycles. The best results are expected for energies that yield the maximum available $n$, leading to nominal rod biases of $+70 \mathrm{~V}$ and $-2 \mathrm{~V}$ for the circular and hyperbolic QMSs respectively, as indicated in Table 1.

\subsubsection{Matching of dynamic range}

Although not directly related to any of the dimensionless quantities, $a, q$, or $\xi$, the instrument sensitivity is an important characteristic of QMS sensors that determines the effective dynamic range of the measured mass spectra signal. Determination of the total instrument sensitivity is a complex task as it will be a function of detector type, efficiency, accumulation time, ion source design, and the partial pressures of measured gases [6]. Therefore, although the measurement timing (Table 1 ) for each sensor differs slightly due to the various requirements of each instrument's interface software, the pressure of the krypton gas inside the ion source was adjusted to obtain similar counting statistics. For the circular rod QMS, the source pressure was set to $1 \times 10^{-5}$ mbar. For the hyperbolic rod sensor, the source pressure was set to $1 \times 10^{-7}$ mbar. These pressures resulted in measurements of $\mathrm{Kr}^{+}$isotopes ranging over approximately 4 orders of magnitude in detected particle flux for both instruments.

\section{Results and discussion}

With these well-matched systems, isotopes of $\mathrm{Kr}^{+}$in the $(m / z)$ range $78-86 \mathrm{amu} / \mathrm{e}$ were measured by each sensor with and without applied higher order auxiliary excitation. These measurements were analyzed in terms of mass resolution, peak variability, and variation with ion energy. Figs. 1 and 2 show the stability regions formed from using $q^{\prime}=0.00$ (i.e., no excitation), $q^{\prime} \approx 0.10$, and $q^{\prime} \approx 0.15$ for the hyperbolic and circular rod sensors, respectively with corresponding voltage scan lines. The voltage scan line of the non-excitation measurements was set such that it passed through the tip of the stability region for a given ion. The voltage scan line of the excitation measurements was set such that it passed through the center of a formed upper stability island. Similar island splitting is shown for both sensors. The hyperbolic rod measurements without excitation created sharper stability boundaries than those of the circular rod sensor, as expected.

The scalar conversion from RF voltage to $\mathrm{m} / \mathrm{z}$ will change depending on instrument operating point. The appropriate conversion from RF voltage to $m / z$ is selected as the factor that aligns the maximum transmission for ${ }^{86} \mathrm{Kr}^{+}$between $\mathrm{m} / \mathrm{z}=85.75 \mathrm{amu} / \mathrm{e}$ and $m / z=86.25 \mathrm{amu} / \mathrm{e}$ for the lowest ion energy setting for each sensor.

\subsection{Mass resolution}

Figs. 3 and 4 show 10 mass scans of the krypton mass range for each QMS with and without applied excitation. The mean of each set of scans is indicated as the solid black line. The $1 \%$ mass resolution $\left(R_{0.01}\right)$ for mass peak ${ }^{86} \mathrm{Kr}^{+}$is indicated for each case, where $R_{0.01}$ is defined as $(\mathrm{m} / \mathrm{z}) / \Delta(\mathrm{m} / \mathrm{z})$ where $\mathrm{m} / \mathrm{z}$ is the measured mass per charge ratio and $\Delta(\mathrm{m} / z)$ is the width of the mass peak that is within $1 \%$ of its maximum transmission value. This value is not computed for all mass peaks as their relative proximity to one another and measured dynamic ranges may not make such a 

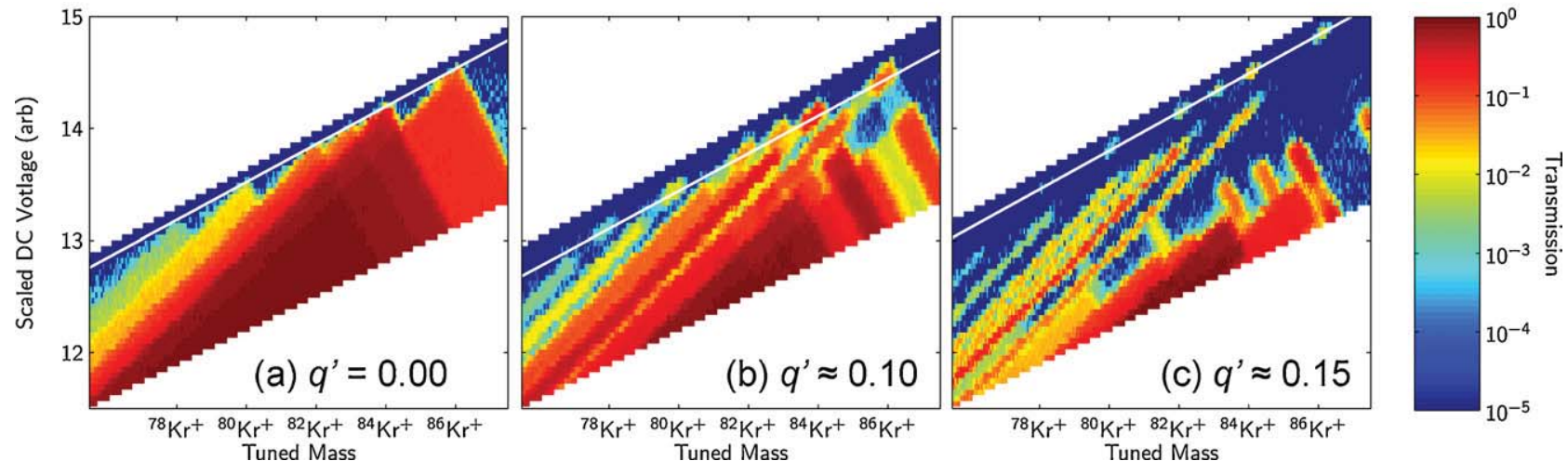

Fig. 1. Stability island formation for the hyperbolic QMS $(n \approx 80)$ with (a) no added excitation $\left(q^{\prime}=0.00\right)$, (b) $q^{\prime} \approx 0.10$, and $(\mathrm{c}) q^{\prime} \approx 0.15$. The scan line used to obtain high resolution measurements is shown on each. For the non-excitation case, i.e., nominal QMS operation, the scan line passes through the tip of the stability region. For the excitation cases, the scan line slope is tuned such that it passes through the center of the formed stability islands.
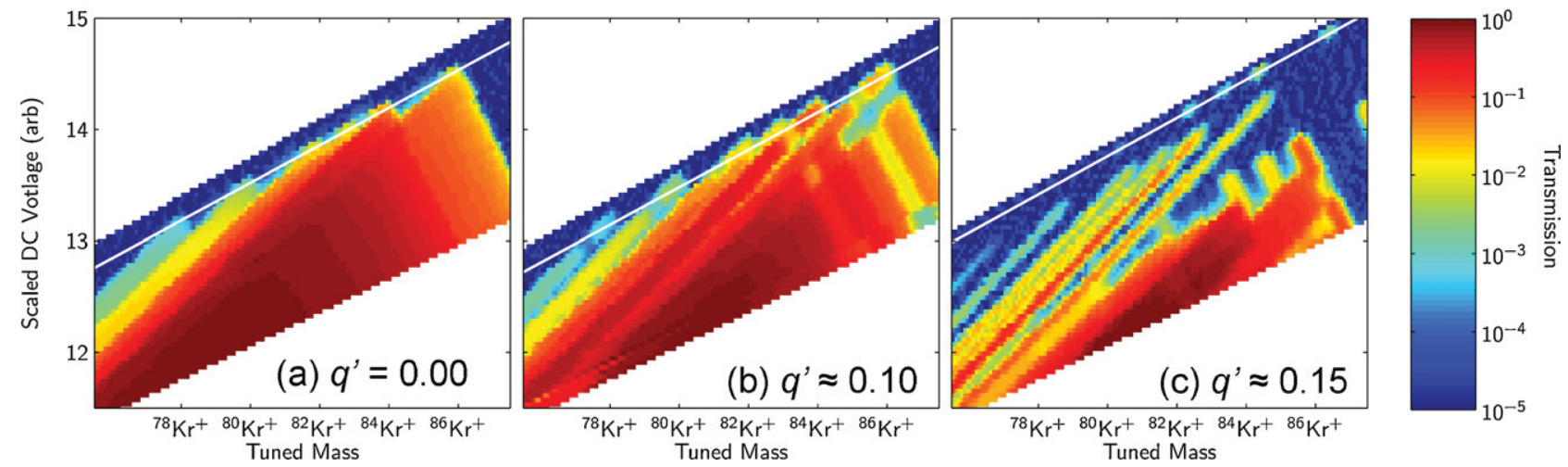

Fig. 2. Same as Fig. 1 but for the circular rod QMS with $n \approx 70$. Similar stability regions are formed but with less sharp boundaries for the non-excitation case, consistent with the low-mass peak tail expected from the circular rod sensor.

computation possible in all cases, specifically for the circular rod set operated without auxiliary excitation. The higher resolution scans were set to have a slope that passed through the very tip of the stability region, at the limit of instrument performance.
With added excitation, the circular rods show a factor of two improvement in the $R_{0.01}$ of ${ }^{86} \mathrm{Kr}^{+}$, consistent with previous measurements of $\mathrm{Ar}^{+}$using a similar experimental setup [16]. For the non-excitation scans, the observed $R_{0.01}$ of the circular rods

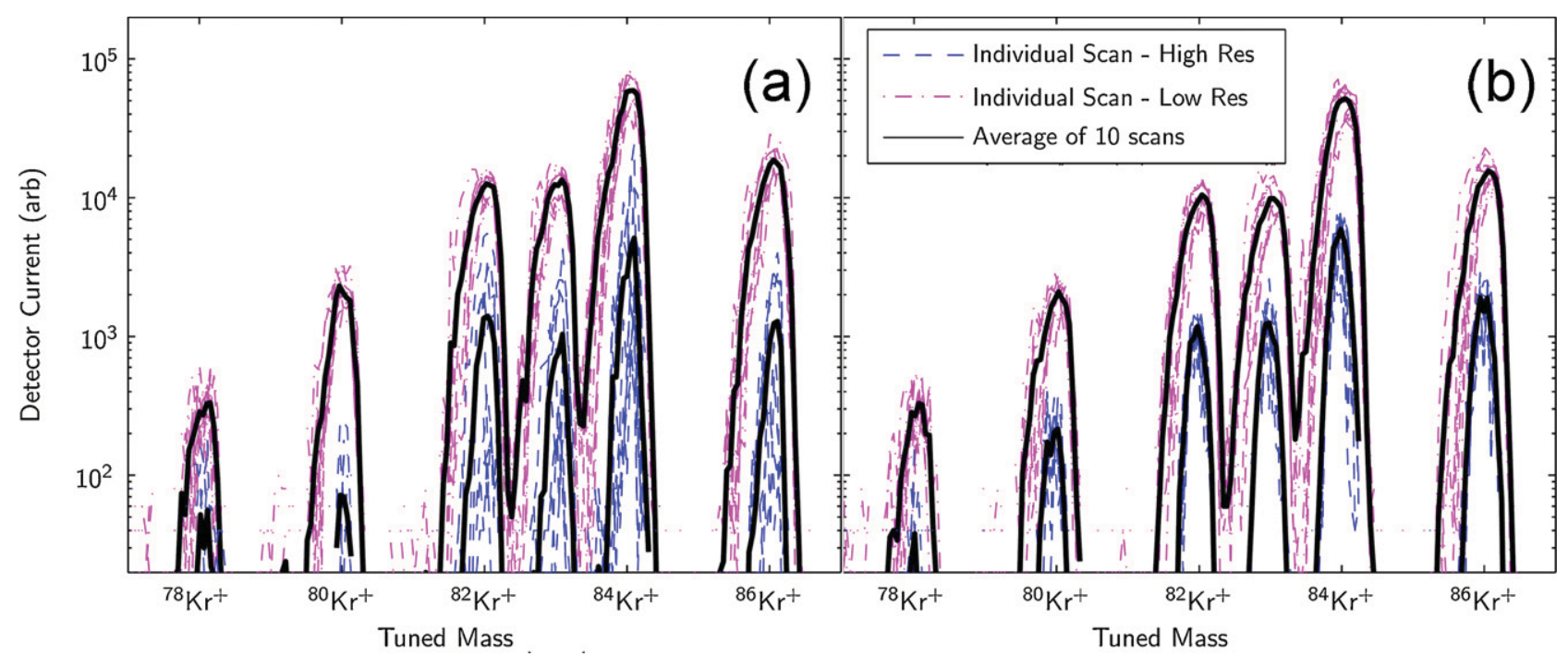

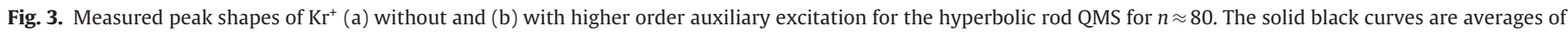
sets of ten successive mass scans, with the pink dash-dotted curves corresponding to a set of lower resolution, stable measurements and the blue dash curves corresponding to the maximum attainable mass resolution with the given test electronics. The lower and higher resolution measurements in (b) use auxiliary excitation levels of $q^{\prime} \approx 0.10$

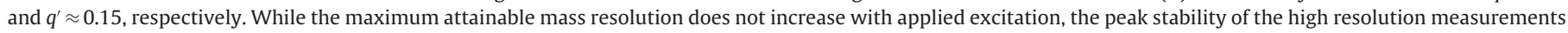
significantly improves. (For interpretation of the references to color in this figure legend, the reader is referred to the web version of the article.) 

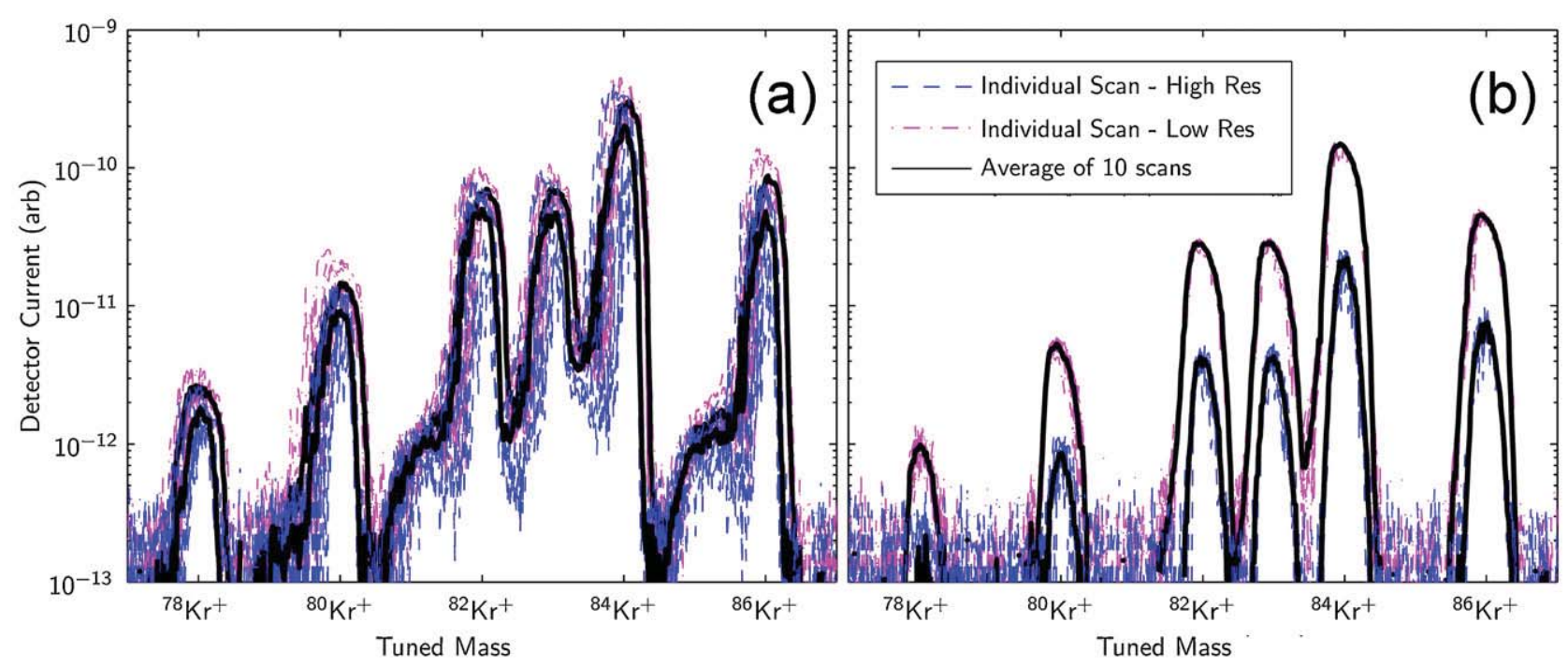

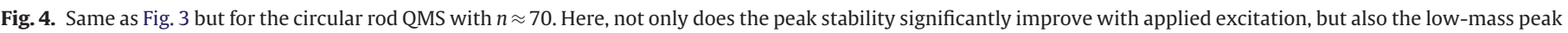
tail characteristic of circular rod sensors is removed, effectively doubling the maximum attainable mass resolution of the instrument. (For interpretation of the references to color in this figure legend, the reader is referred to the web version of the article.)

is reduced by the low mass peak tail, which arises as a consequence of imperfections in the quadrupole electric field resulting from non-ideal rod geometry. Auxiliary excitation techniques increase rejection efficiency of unwanted ions near these boundaries, removing this peak tail. However, this tailing effect is not observed in the hyperbolic QMS measured peaks, even for the nonexcitation case. Consequently, the $R_{0.01}$ value for the hyperbolic rod set remains unchanged with added excitation, though the highest possible mass resolution for the hyperbolic rod system was slightly larger than that of the circular rod system. These results are consistent with predictions from numerical simulations $[17,18]$ that show for ideal quadrupole geometry the overall mass resolution for a particular ion transmission is only expected to improve for higher $(R \sim 1000)$ resolution measurements.

\subsection{Peak variability and stability}

Electronics noise causes small shifts in operating point during a mass scan. In the traditional mode of quadrupole operation, for lower resolution scans, these shifts will not create large changes in mass peak shapes. However, for higher resolution scans, when operating scan lines through the tip of stability regions, the mass peak shapes become very sensitive to small changes in operating point. This effect can be observed in the non-excitation peaks of both the hyperbolic and circular rod systems, which become increasingly variable and unstable at high resolution. Scan line operation through the center of an upper stability island, however, mitigates this effect. These islands represent large, symmetric areas of almost constant transmission and resolution. Therefore, small shifts in operating point do not result in significant changes to the mass peak shapes, resulting in more stable peak shapes for both rod geometries, as shown in Figs. 3 and 4.

The accuracy of recovered isotopic ratios of krypton is used to quantify this variability. The $10 \%$ transmission, $T_{0.1}$, is the average transmission between the two points defining the $10 \%$ resolution of a mass peak. This value was determined for each of the mass peaks corresponding to singly charged krypton isotopes, ${ }^{78} \mathrm{Kr}^{+},{ }^{80} \mathrm{Kr}^{+}$, ${ }^{82} \mathrm{Kr}^{+},{ }^{83} \mathrm{Kr}^{+},{ }^{84} \mathrm{Kr}^{+}$, and ${ }^{86} \mathrm{Kr}^{+}$, and was normalized by the total $T_{0.1}$ for all measured masses, giving the measured relative abundance of each isotope for each scan. The mean of the derived relative abundances of the 10 scans are shown in Fig. 5 and are compared to the standard literature values [19]. The error bars in Fig. 5 indicate the standard deviation of the 10 scans, a proxy for the variability of the mass peak shapes. The measurements of ${ }^{78} \mathrm{Kr}^{+}$had a very low signal to noise ratio for both the circular rod and hyperbolic rod higher resolution scans, resulting in misleadingly high calculations of variability. For other peaks, counting statistics result in pointwise errors on the order of $10 \%$ of the peak value. The transmission for both the excitation and non-excitation curves are similar, so counting statistical error would have a similar effect on both sets of peaks, indicating that the increase in stability is due to the use of auxiliary excitation. The $1 \%$ transmission, $T_{0.01}$, was not used for this analysis due to insufficient separation of mass peaks for the case of circular rods operated without excitation.

The extremely high variability of the non-excitation high resolution hyperbolic rod mass scans is likely a result of operation at a sharp tip of the stability region, where small changes in operating point create drastic changes in transmission combined with the limitations of our interfaced electronics as listed above. This effect is likely less pronounced in the circular rod system since the tip of this region is 'blunted' by non-linear resonant effects [20].

While the average measured abundances do not significantly change with excitation, the variability of each scan is significantly reduced for high-resolution operation with applied excitation. This reduction in variability directly translates into a shorter required scanning time for instrument high resolution scans. For example, the average high resolution variability of the hyperbolic rods with applied excitation was 6 times lower than the non-excitation case, leading to a 6 times shorter required scan time. Likewise, the circular rod high resolution scanning time could be reduced by a factor of 2 . This increased peak stability is consistent with previous experimental work, as well as predictions from numerical simulations [16,17].

\subsection{Ion rejection efficiency}

From both numerical simulations [18] and previous experimental work [13], the number of RF cycles required for sufficient rejection of undesired ions is expected to decrease when operating with applied excitation, i.e., the rejection efficiency should increase, and consequently the attainable resolution for a given ion energy [6]. This improvement is likely the reason for the improved maximum attainable mass resolution in Section 3.1 for the circular rod 


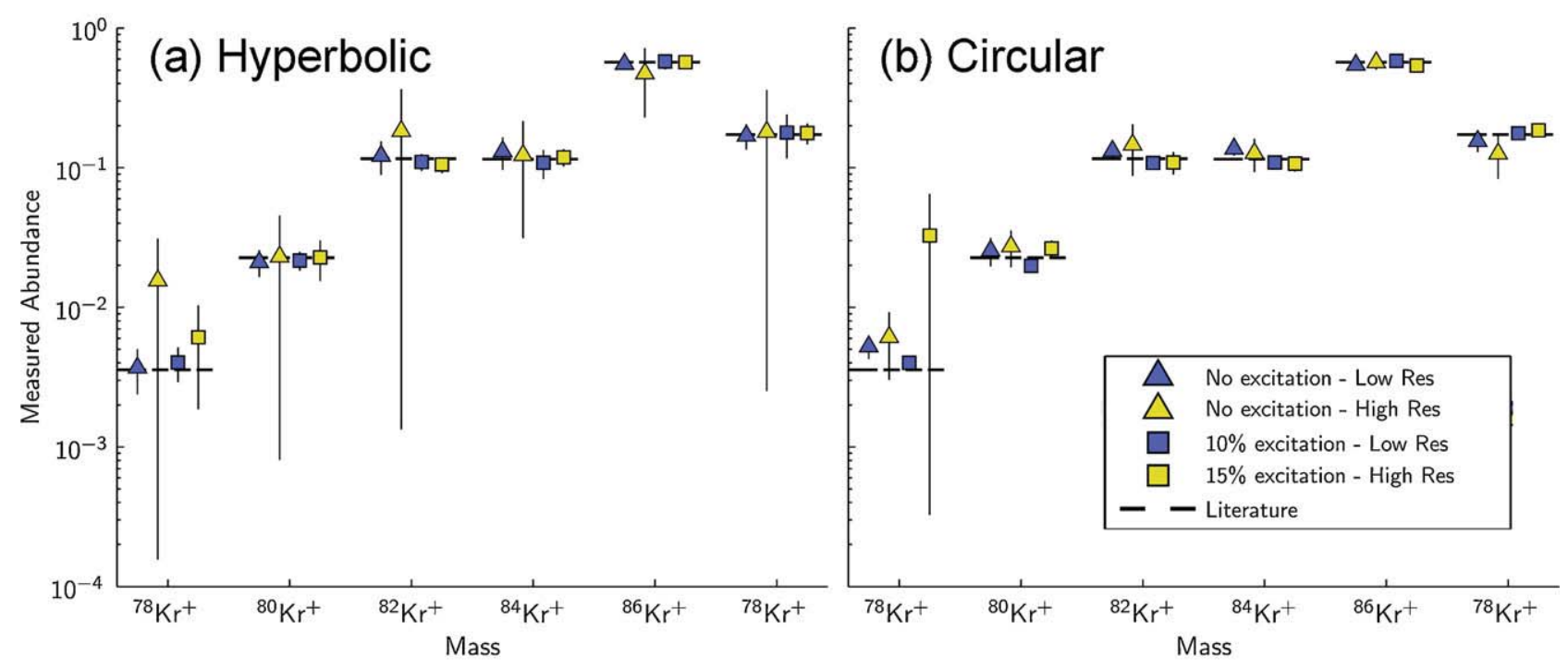

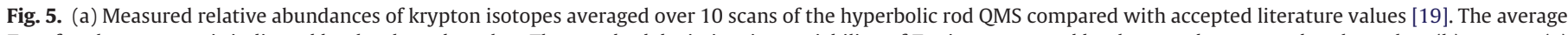

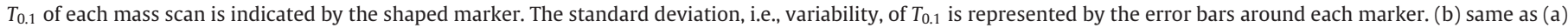
but for the circular rod QMS. For all cases, the peak variability at the limit of the test electronics (high resolution modes) significantly improves with applied excitation.

quadrupole. The maximum attainable resolution was achieved at the limit of the test electronics. In order to characterize the effect of excitation on mass peaks independent of electronics precision effects, the rejection efficiency is measured using the lower resolution instrument settings from Section 3.1 (i.e., the pink dash-dotted curves from Figs. 3 and 4).

As discussed in Section 2.1.2, the number of RF cycles an ion spends traversing the rods can be adjusted by changing the QMS rod bias. The ranges of rod biases from Section 2.1.2 were scanned for each system with and without applied excitation. Figs. 6 and 7 show the effect of changing the rod bias on mass peak shapes for each sensor. For reduced $n$, for both the hyperbolic and circular rod systems, significant peak tailing occurs on the low mass side of the $\mathrm{Kr}^{+}$mass peaks. However, this effect is less pronounced for operation with applied excitation.

Because of insufficient separation between adjacent mass peaks, it is difficult to directly calculate the changes in mass resolution of $\mathrm{Kr}^{+}$without fitting models of the mass peaks to the observed spectra. Instead, to quantify these effects, the crosstalk of ${ }^{85} \mathrm{Kr}^{+}$ relative to the transmission of the true ${ }^{86} \mathrm{Kr}^{+}$peak is calculated. ${ }^{85} \mathrm{Kr}^{+}$does not correspond to any known naturally occurring stable isotope of krypton [19]. Therefore any transmission between $m / z=84.75 \mathrm{amu} / \mathrm{e}$ and $m / z=85.25 \mathrm{amu} / \mathrm{e}$ is considered to be mass crosstalk. This transmission, $T^{85}$, is normalized by the transmission of the adjacent peak, ${ }^{86} \mathrm{Kr}^{+}$, between $\mathrm{m} / z=85.75 \mathrm{amu} / \mathrm{e}$ and $m / z=86.25 \mathrm{amu} / \mathrm{e}$, and plotted in Fig. 8 for both rod sets.

The ${ }^{85} \mathrm{Kr}^{+}$mass crosstalk in the non-excitation circular rod measurements is high due to the significant low mass peak tail that is present even for large values of $n$. This tail is eliminated with the use of auxiliary excitation for all ion energies. Consequently, the relative reduction of mass crosstalk due to auxiliary excitation (about an order of magnitude for all $n$ ) is highest for the circular rod system. However, even for the hyperbolic rod set, the relative reduction when using auxiliary excitation is at least a factor of two and up to a factor of 20 .
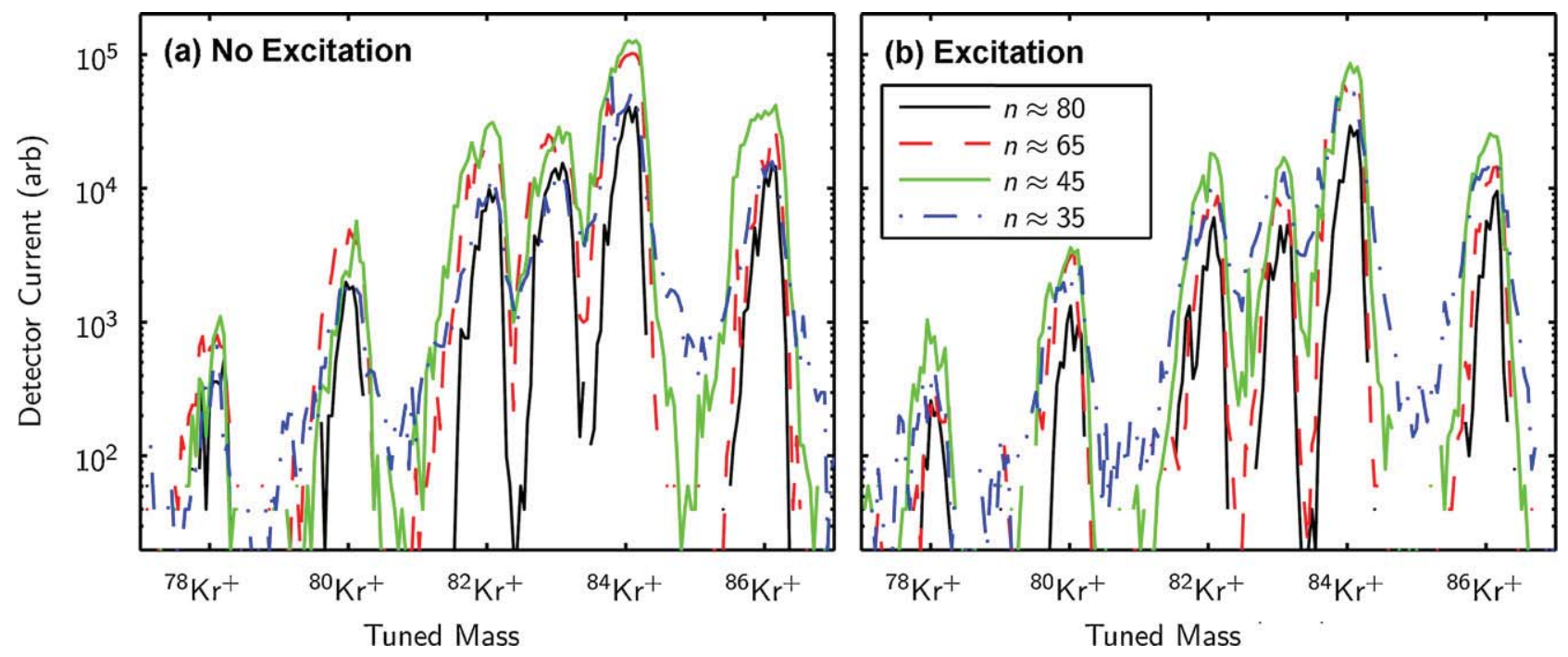

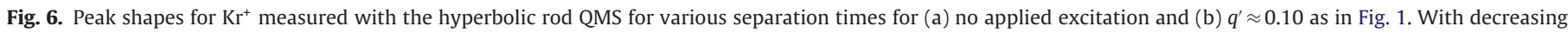
number of RF cycles, $n$, the measured mass peaks exhibit tailing on both the low-mass and high-mass sides. Such tailing is mitigated with applied excitation. 

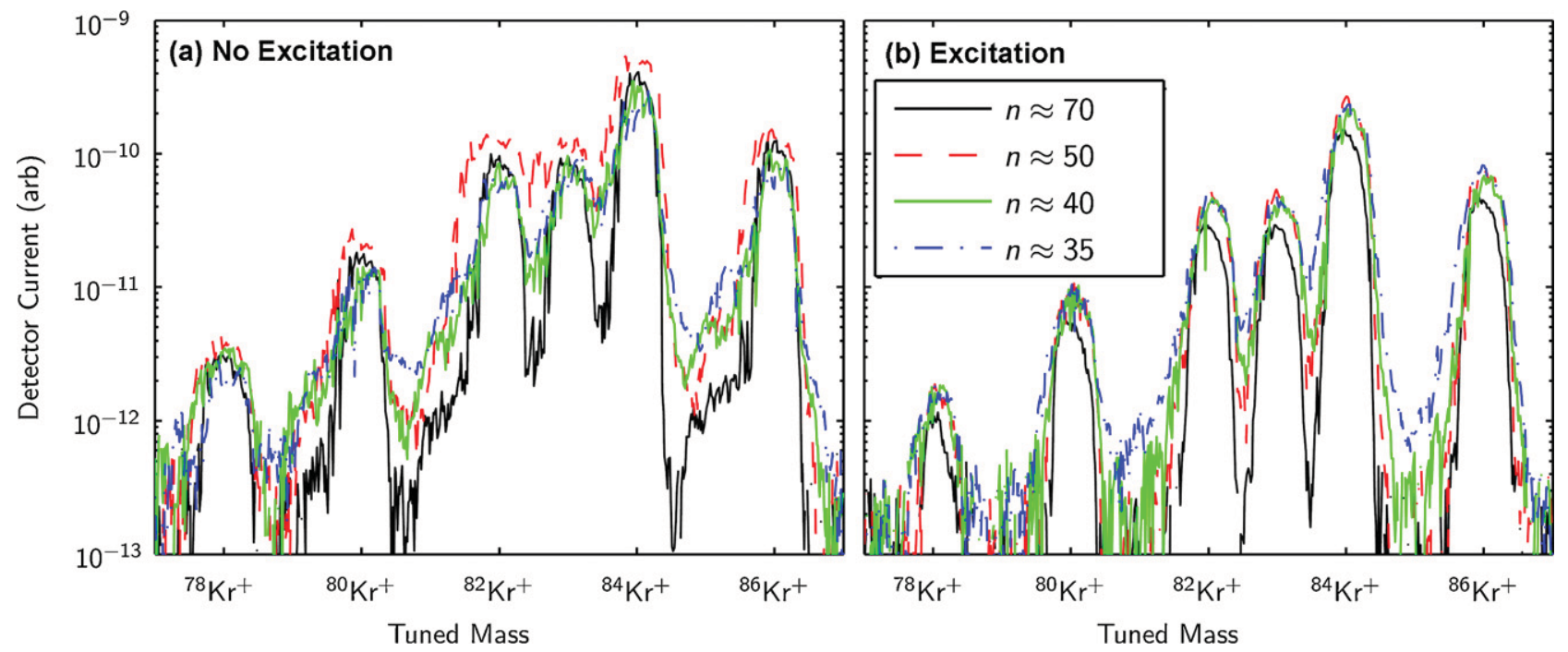

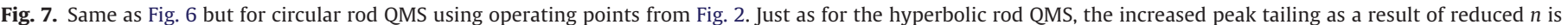
mitigated with applied excitation, including the peak tail that appears at all $n$-values as a consequence of circular rod geometry.
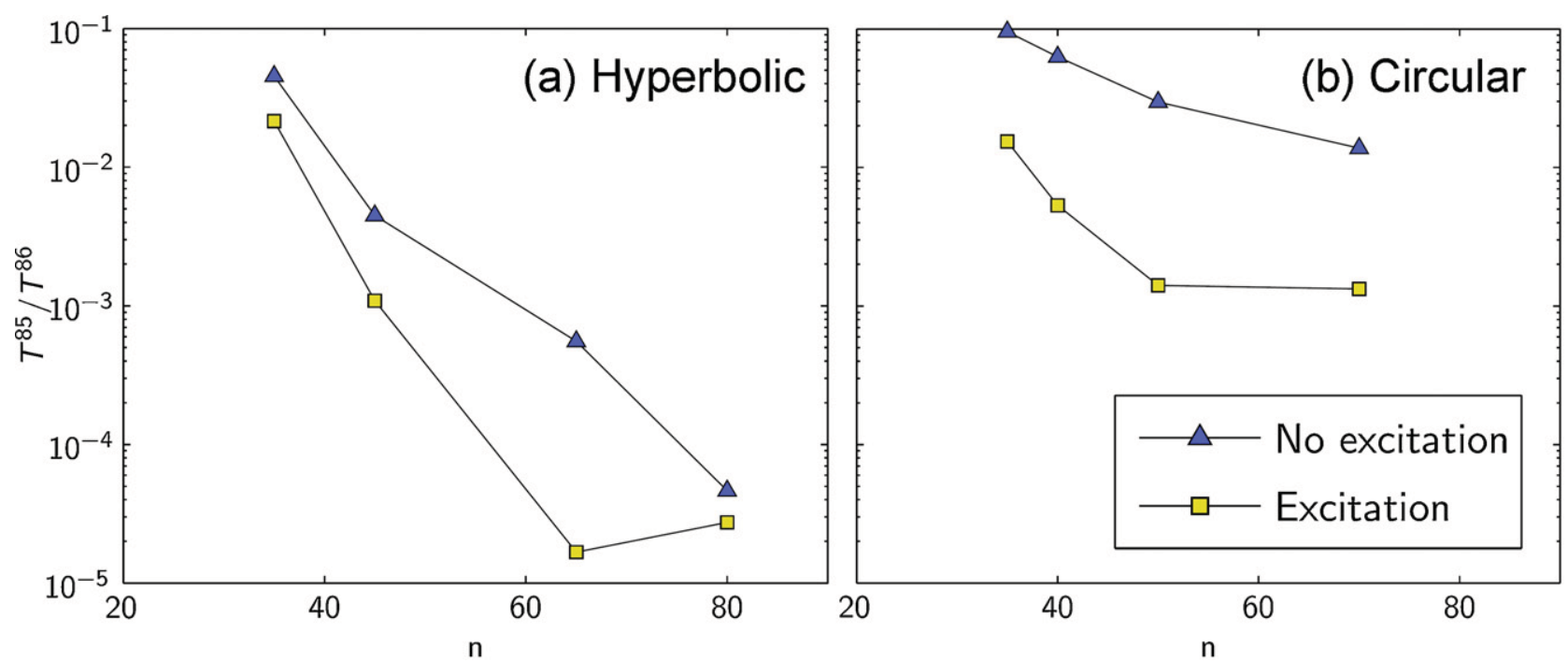

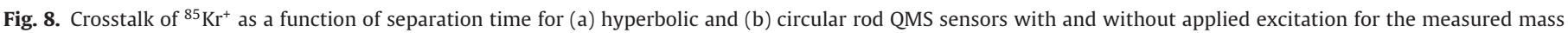
peaks from Figs. 6 and 7. The crosstalk is reduced by factors of 2-20 for both measurement geometries.

As seen in Fig. 8, with added excitation, both QMS sensors require only approximately $67 \%$ of the $n$-value to obtain performance comparable to the non-excitation cases. Lowering $n$ has two major implications for QMS sensors: (1) the quadrupole rods themselves can be shortened to create a smaller, lighter instrument without sacrificing performance and (2) higher energy ions may be analyzed, simultaneously relaxing design constraints on the ion source and creating an increased flux of particles at the detector, thereby increasing instrument sensitivity and enabling shorter measurement times.

\section{Conclusions}

High order auxiliary excitation significantly improved the performance of the Pfeiffer QMG422 commercial circular rod quadrupole in all areas, doubling the maximum attainable $R_{0.01}$ for the given test electronics, providing a two-fold increase in peak stability and variability, and improving ion rejection efficiency by an order of magnitude. Although the maximum attainable $R_{0.01}$ of the MSL/SAM hyperbolic QMS system did not improve with applied auxiliary excitation, six and two-fold improvements were obtained for peak stability and ion rejection efficiency, respectively. These results are all consistent with previous experimental work and numerical simulations related to auxiliary excitation techniques. Consequently, the results of these simulations, specifically the improved predicted performance of hyperbolic rod sensors for high resolution modes are expected to hold for sensors employing higher precision electronics than those used in this study. One of the most significant improvements for ideal geometry sensors is that of enhanced peak stability obtained from operating voltage scan lines through the center of formed upper stability islands. As of this work, the only power efficient way of implementing such a technique at high resolutions is to use higher order auxiliary excitation in conjunction with a multiresonant tank circuit.

Based on the derived resolution of these measurements, it is difficult to distinguish between mass peaks obtained from different rod geometries when auxiliary excitation is applied. This similarity in observed performance is even more remarkable when considering the quality of the respective ion sources used for each sensor. The hyperbolic rod ion source is one of the most precise and stable 
of its kind, with the circular rod QMS using a standard, commercially available ion source with a comparatively wide entrance aperture and ion beam size, factors that significantly affect the performance of the sensor in its traditional mode of operation. Higher order auxiliary excitation techniques are clearly a powerful tool for mitigating effects of imperfections in a quadrupole system, whether they arise from fringing fields, non-linear resonances, or wide ion energy ranges. These potential improvements to circular rod systems could have tremendous impact on the next generation of quadrupole sensors, as circular rod QMS sensors could be created that are suitable for high resolution spaceborne or laboratory applications. We note, however, that the highest attainable mass resolution was measured on the hyperbolic rods operating with auxiliary excitation. We therefore suggest that these techniques could be used to improve the stability and performance of existing hyperbolic sensors, extending their practical mass ranges, and enabling their use for future planetary and cometary missions.

\section{Acknowledgments}

The authors would like to acknowledge Eric Raaen, Heather Franz, and Mike Barciniak for their assistance and support with interfacing to and operating the MSL/SAM test bed system. This work was supported by the NASA Planetary Instrument Definition and Development NNX08A005G and NASA Graduate Student Research Program NNX09AL50H grants. The University of Michigan is pursuing patent protection for the presented intellectual property and is seeking commercialization partners to help bring the technology to market.

\section{References}

[1] R.E. March, J.F.J. Todd, Quadrupole Ion Trap Mass Spectrometry, John Wiley, and Sons, Inc., Hoboken, NJ, 2005.

[2] J.H. Waite, W.S. Lewis, W.T. Kasprzak, V.G. Anicich, B.P. Block, T.E. Cravens, G.G. Fletcher, W.H. Ip, J.G. Luhmann, R.L. Mcnutt, H.B. Niemann, J.K. Parejko, J.E. Richards, R.L. Thorpe, E.M. Walter, R.V. Yelle, The Cassini Ion and Neutral Mass Spectrometer (INMS) investigation, Space Sci. Rev. 114 (2004) 113.

[3] H.B. Niemann, D.N. Harpold, S.K. Atreya, G.R. Carignan, D.M. Hunten, T.C. Owen, Galileo probe mass spectrometer experiment, Space Sci. Rev. 60 (1992) 111.
[4] H.B. Niemann, J.R. Booth, J.E. Cooley, R.E. Hartle, W.T. Kasprzak, N.W. Spencer, S.H. Way, D.M. Hunten, G.R. Carignan, Pioneer Venus orbiter neutral gas mass spectrometer experiment, IEEE Trans. Geosci. Remote Sens. 18 (1980) 60.

[5] H.B. Niemann, S. Atreya, S.J. Bauer, K. Biemann, B. Block, G. Carignan, T. Donahue, L. Frost, D. Gautier, D. Harpold, D. Hunten, G. Israel, J. Lunine, K. Mauersberger, T. Owen, F. Raulin, J. Richards, S. Way, The gas chromatograph mass spectrometer aboard Huygens, in: A. Wilson (Ed.), Huygens: Science, Payload and Mission, ESA, Paris, 1997, p. 85

[6] P.H. Dawson, in: H. Peter, Dawson (Eds.), Quadrupole Mass Spectrometry and its Applications, Elsevier, New York, 1976.

[7] D.J. Douglas, N.V. Konenkov, Influence of the 6th and 10th spatial harmonics on, the peak shape of a quadrupole mass filter with round rods, Rapid Commun. Mass Spectrom. 16 (2002) 1425.

[8] P.R. Mahaffy, C.R. Webster, M. Cabane, P.G. Conrad, P. Coll, S.K. Atreya, et al. The sample analysis at MARS investigation and instrument suite, Space Sci Rev., submitted for publication.

[9] M.J.S. Belton, New Frontiers in the Solar System - An Integrated Exploration, Strategy, The National Academies, Washington, DC, 2003.

[10] P. Marchard, P. Marmet, Opération D'un Filtre de Masse Quadrupolaire en, Condition Optimum, Can. J. Phys. 42 (1964).

[11] G. Devant, P. Fercocq, G. Lepetit, O. Maulat, Procede d'alimentation en tension, des spectrographs de mass du type quadripolaire, French patent 2,620,568 (1989).

[12] K. Miseki, Quadrupole mass spectrometer, US patent 5,227,629 (1993).

[13] N.V. Konenkov, L.M. Cousins, V.I. Baranov, M. Yu Sudakov, Quadrupole, mass filter operation with operation with auxiliary quadrupolar excitation: theory and experiment, Int. J. Mass Spectrom. 208 (2001) 17.

[14] X. Zhao, Z. Xiao, D.J. Douglas, Overcoming field imperfections of quadrupole mass filters with mass filters with mass analysis in islands of stability, Anal. Chem. 81 (2009) 5806.

[15] X. Zhao, Z. Xiao, D.J. Douglas, Mass analysis with islands of stability with linear quadrupole incorporating higher order multipole fields, J. Am. Soc. Mass Spectrom. 21 (3) (2010) 393.

[16] D.J. Gershman, B.P. Block, M. Rubin, M. Benna, P.R. Mahaffy, T.H. Zurbuchen, Higher order parametric excitation modes for spaceborne mass spectrometers, Rev. Sci. Instrum. 82 (2011) 125109, http://dx.doi.org/10.1063/1.3669781.

[17] D.J. Gershman, M. Rubin, B.P. Block, M. Benna, P.R. Mahaffy, T.H. Zurbuchen, Enabling the next generation of spaceborne quadrupole mass spectrometers, in: ASCE Conf. Proc. Earth and Space, in press, http://content.asce.org/ conferences/earthspace2012/index.html.

[18] N.V. Konenkov, A. Korolkov, M. Machmudov, Upper stability island of the, quadrupole mass filter with amplitude modulation of the applied voltages, J. Am. Soc. Mass Spectrom. 16 (2005) 379.

[19] N.E. Holden, Table of the isotopes, in: D.R. Lide (Ed.), CRC Handbook of Chemistry and Physics, 85th ed., CRC Press, 2004, ISBN 978-0849304859, Section 11.

[20] J. Schulte, P.V. Shevchenko, A.V. Radchik, Nonlinear field effects in quadrupole mass filters, Rev. Sci. Instrum. 70 (1999) 3566, http://dx.doi.org/10.1063/1.1149960. 